\title{
FLUCONAZOLE SUSCEPTIBILITY TESTING OF CANDIDA SPECIES BY DISC DIFFUSION AND AGAR DILUTION METHOD
}

Supriya Tankhiwale, Sunita Gajbhiye, Rajaram Powar

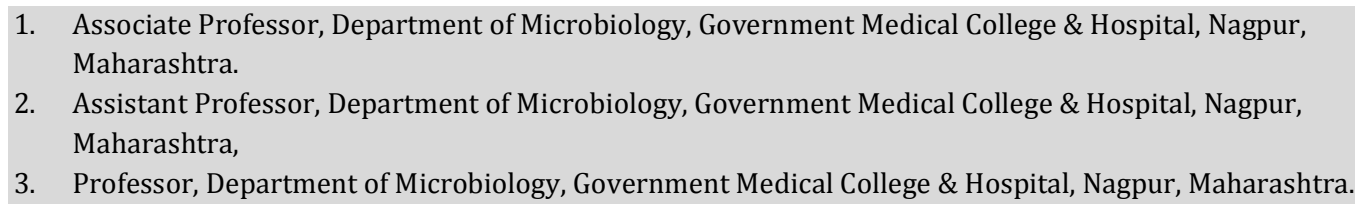

\section{CORRESPONDING AUTHOR}

Dr. Sunita Gajbhiye

OASIS, plot no 11, Anand Nagar, Atrey layout, Nagpur-440022, Maharashtra, India

E-mail: sunitarajgaj@yahoo.com, Ph: 00919325339668.

ABSTRACT: PURPOSE: Fungal infections with candida species are an important cause of morbidity and mortality ${ }^{1}$. Situation is further worsened by increasing resistance to antifungal drugs ${ }^{2}$.The objective of this study was to evaluate antifungal susceptibility pattern of Candida species to fluconazole by disc diffusion and agar dilution method and compare these two methods as far as fluconazole is concerned. MATERIAL \& METHODS: Between January 2006 to December 2006, 119 Candida species were isolated from 225 sputum samples of patients of pulmonary tuberculosis and immunocomprised patients. RESULTS: Of the 119 Candida isolates $33(27.73 \%)$ were Candida albicans, 32 (26.89\%) were Candida gullermondi, 27 (22.68\%) Candida tropicalis, 14 (11.76\%) Candida pseudotropicalis, 7 (5.88\%) Candida parapsilosis and 6 (5.04\%) were Candida krusei. The Candida isolates were tested for fluconazole by disc diffusion method on Muller hinton agar with 2\% glucose and 0.5 microgram of methylene blue ${ }^{3}$.Amongst the 119 Candida isolates, 17 (14.28\%) isolates were resistant to fluconazole by disc diffusion method. MIC done by agar dilution method using CLSI guidelines 4. Candida isolates showed growth of 15 (88.23\%) Candida strains upto 8 microgram / ml with $80 \%$ inhibition of growth whereas $2(1.7 \%)$ isolates showed MIC upto 64 microgram/ ml. CONCLUSIONS: Candida albicans though a common species other species were isolated in significant number. The results of disc diffusion for fluconazole do not correlate with agar dilution method. To cater the need of resource constrained laboratories, it is important to have results that correspond to the clinical outcome of antifungal treatment and show co-relation to those obtained with the reference method as recommended by CLSI guidelines and also be reproducible ${ }^{5}$. Further critical studies are needed.

KEYWORDS: Candida, Fluconazole, MIC, Agar dilution method.

INTRODUCTION: Fungal infections have dramatically increased in recent years along with the increase in drug resistant isolates ${ }^{1}$. The last few decades have seen a steady increase in the incidence of fungal infections especially Candida species. Prolonged antibiotic therapy, invasive therapeutic procedures, radiotherapy and AIDS pandemic have all contributed to the rise in these infections. Other causes include recipients of solid organ transplant and cytotoxic chemotherapy ${ }^{3}$.Candida species are the most common pathogens as far as fungal isolates are concerned. 
Candidiasis is the most common fungal disease with a wide spectrum of clinical presentation.The infection may be acute or chronic, superficial or deep involving various organs $^{3}$. Since the Candida species are normal commensals of human skin, throat, gastro intestinal tract, female genital tract and urine of patients with indwelling catheters, its pathogenic role is to be confirmed by repeated isolation. The present study is undertaken with following objectives,

a) To study the antifungal sensitivity pattern of Candida isolates using disc diffusion method

b) To study the MIC of fluconazole by using agar dilution method

MATERIALS AND METHODS:- Sputum samples from patients with chronic lung diseases with or without immunocompromised state were collected. Patients with upper respiratory tract infection and also in patients where Candida species were not isolated for more than two times were excluded from the study. Early morning first sputum sample was collected after thorough rinsing of mouth in sterile wide mouth container and transported immediately to the microbiology laboratory. Sputum samples were processed for
a) Gram stain
b) $\mathrm{KOH}$ preparation
c) Culture on Sabarauds Dextrose Agar(SDA)

The Sputum samples showing pseudohyphae, large number of budding yeast cells were included in the study. Culture on SDA showing more than 25-30 colonies or confluent heavy growth of Candida was considered as pathogenic. A second sputum sample was collected for confirmation and re-isolation. The Candida isolates were subjected to germ tube test and cultured on corn meal agar. Depending on chlamydospore formation, the species were identified. Confirmation of the species was done by sugar assimilation test ${ }^{6}$. Antifungal susceptibility testing by disc diffusion method for fluconazole was done using fluconazole disc from Hi-media laboratories on Muller-Hinton agar with 2\% glucose and 0.5 microgram methylene blue. Other drugs tested were- Ketoconazole, Clotrimazole, Nystatin and Amphotericin- B. The standard strain used as control was Candida albicans ATCC 90028 and the zone sizes ${ }^{7}$ were noted as follows:

- Candida albicans - $28.8 \mathrm{~mm} \pm 6.5 \mathrm{~mm}$

- Candida tropicalis- $27 \mathrm{~mm} \pm 8 \mathrm{~mm}$

- Candida gullermondi-24.7mm $\pm 7.2 \mathrm{~mm}$

- Candida parapsilosis- $32.8 \mathrm{~mm} \pm 7.4 \mathrm{~mm}$

- Candida kruzei-18mm $\pm 8.5 \mathrm{~mm}$

The minimum inhibitory concentration for fluconazole was done by agar dilution method as per the CLSI guidelines. The MIC reading was defined as the lowest concentration inhibiting at least $50 \%$ of the growth. The isolates tested for fluconazole showing MIC of

- 8 microgram / $\mathrm{ml}$ were classified as susceptible,

- Those with MIC more than 64 microgram / $\mathrm{ml}$ were labeled as resistant and

- The isolates with MIC between 16 - 32 were termed as susceptible dose dependent. ${ }^{7}$ 


\section{OBSERVATIONS}

TABLE NO. I $\quad \mathrm{n}=119$

\begin{tabular}{|l|l|l|l|}
\hline S. NO. & CANDIDA SPECIES & NO. & $\%$ \\
\hline 1 & C. albicans & 33 & 27.73 \\
\hline 2 & C. gullermondi & 32 & 26.89 \\
\hline 3 & C. tropicalis & 27 & 22.68 \\
\hline 4 & C. pseudotropicalis & 14 & 11.76 \\
\hline 5 & C. parapsilosis & 07 & 5.88 \\
\hline 6 & C. krusei & 06 & 5.04 \\
\hline
\end{tabular}

Of the 225 sputum samples received, 119 candida species were isolated. Candida albicans was the most commonly isolated species followed by Candida gullermondi and Candida tropicalis

TABLE NO.2

Sensitivity pattern of Candida species to fluconazole by disc diffusion method ( $n=119)$

\begin{tabular}{|l|l|l|l|}
\hline S.no & Candida species & Sensitive & Resistant \\
\hline 1 & C. albicans ( $n=33)$ & 31 & 02 \\
\hline 2 & C. gullermondi (n=32) & 29 & 03 \\
\hline 3 & C. tropicalis (n=27) & 26 & 01 \\
\hline 4 & C. pseudotropicalis ( $n=14)$ & 11 & 03 \\
\hline 5 & C. parapsilosis (n=7) & 05 & 02 \\
\hline 6 & C. krusei $(\mathrm{n}=6)$ & 00 & 06 \\
\hline & Total & 102 & 17 \\
\hline
\end{tabular}

From the above table it is evident that maximum sensitivity to fluconazole was seen with Candida albicans. Non - albicans Candida species also showed a similar pattern of sensitivity with fluconazole. Candida krusei is inherently resistant to fluconazole, hence all Candida krusei isolates showed resistance to the drug . 
TABLE NO.3

MIC of Candida species to fluconazole by agar dilution method

\begin{tabular}{|c|c|c|c|c|c|}
\hline \multirow[t]{2}{*}{ S. no } & \multirow[t]{2}{*}{$\begin{array}{ll}\text { Candida species } \quad(n=17)\end{array}$} & \multicolumn{4}{|c|}{ Minimum Inhibitory Concentration } \\
\hline & & 8 & 16 & 32 & 64 \\
\hline 1 & C. albicans & 01 & & & 01 \\
\hline 2 & C. gullermondi & 02 & & & 01 \\
\hline 3 & C. tropicalis & 01 & & & \\
\hline 4 & C. pseudotropicalis $\quad(n=3)$ & 03 & & & \\
\hline 5 & C. parapsilosis & 02 & & & \\
\hline 6 & C. krusei & 06 & & & \\
\hline & Total & 15 & & & 02 \\
\hline
\end{tabular}

MIC was done for fluconazole for all species of Candida. All the strains which were sensitive by disc diffusion method were also sensitive by agar dilution method. their MIC ranged from 0-8 microgram / ml .

\section{TABLE NO.5}

Comparison between disc diffusion method and agar dilution method for fluconazole susceptibility of candida isolates

\begin{tabular}{|l|l|l|l|}
\hline \multirow{2}{*}{ Agar dilution method } & \multicolumn{2}{|l|}{ Disc diffusion method } & Total \\
\cline { 2 - 4 } & Sensitive & Resistant & \\
\hline Sensitive & 102 & 15 & 117 \\
\hline Resistant & 0 & 02 & 02 \\
\hline Total & 102 & 17 & 119 \\
\hline
\end{tabular}

Results of fluconazole resistance differ in disc diffusion method and agar dilution method. Their sensitivity matches with each other.

Positive predictive value (ppv) of sensitive test by disc diffusion method $=100 \%$ Positive predictive value (ppv) of resistant test by disc diffusion method $=11.76 \%$

DISCUSSION: The present study was carried out to determine the in- vitro susceptibility pattern of Candida isolates to fluconazole by disc diffusion method and MIC by agar dilution method .out of 225 sputum samples, 119 Candida species were isolated .These isolates were tested for 
fluconazole susceptibility using fluconazole disc on Mueller Hinton agar with $2 \%$ glucose and 0.5 microgram methylene blue ${ }^{6}$. In this study, Candida albicans though a common isolate, other non albicans species were isolated in significant number. The findings are comparable with a study by Khan .S. et al 2006. Fluconazole susceptibility testing using disc diffusion method showed 17 (14.28 \% ) strains to be resistant to the drug, whereas MIC of these strains by agar dilution method showed only $2(1.7 \%)$ strains to be resistant . This is comparable with a study by Leibowitz L D ${ }^{8}$ et al and Rex JH, Rinaldi MG et al.

The co- relation between these two methods is not comparable. Statistical analysis with respect to sensitivity showed that results of disc diffusion and agar dilution match with each other, however the results of resistance differ. To cater the need of resource constrained laboratories, it is important to have an antifungal susceptibility testing method that gives results which correspond to clinical outcome of antifungal treatment, show co- relation with reference method and be reproducible. The major sources of susceptibility test variation for fluconazole and other azoles in vitro have been reported to be $\mathrm{pH}$, the composition of the test medium, inoculums size, temperature and duration of incubation. In addition, partial inhibition of fungal growth in vitro often takes place over a range of fluconazole concentration which can make end point determinations both difficult and subjective ${ }^{9}$.

Further critical studies with large sample size needed.

\section{REFERENCES:}

1. Jiun-Ling Wang, Shan-Chwen Chang, Po-Ren Hsuch, Yee-Chun Chen. In vitro antifungal susceptibility testing of candida blood isolates and evaluation of e- method. J. micro. Immunol. Inf . 2004 ; 37; 335-342.

2. Rex JH, Rinaldi MG, Pfaller MA. Resistance of Candida species to fluconazole. Antimicrob. Agents Chemother 1995 ;39:1-8.

3. Lee SC, Fung CP, Lee N, See LC, Huang JS, Tsai CJ. Fluconazole disc diffusion test with methylene blue and glucose enriched Muller-Hinton agar for determining susceptibility of Candida species. J Clin Microbiol 2001;39:1615-7.

4. National Committee for Clinical Laboratory Standards. Method for antifungal disc diffusion susceptibility testing of yeast; proposed guideline. NCCLS document M44-P, Wayne PA: National committee for Clinical Laboratory Standards Press; 2003.

5. Khan S, Singhal S, Mathur T, Upadhyay DJ, Rattan A. Antifungal susceptibility testing method for resource constrained laboratories. IJMM 2006;Vol.24;No.3:171-176.

6. L.J.R Milne in Fungi in Mackie and McCartney's Practical Medical Microbiology 14th edition, J.Gerald Collee, Marmion BP, Editors Churchill Livingston 699-700.

7. Rex JH, Pfaller MA, Walsh TJ, Chaturvedi V, Espinel-Ingroff A, Ghannoum MA. Antifungal susceptibility testing: Practical aspects and current challenges. Clin Microbiol Rev 2001;14:643-58.

8. Leibowitz LD, Ashbee HR, Evans EG, Chong Y, Mallatova N, Zaidi M. Global Antifungal Surveillance Group. A two year global evaluation of Candida species to fluconazole disc diffusion. Diagn. Microbiol Inf. Dis. 2001;40:27-33

9. MA Pfaller, B Dupont, GS Kobayashi, J Muller, MG Renaldi, A Espinel-Ingroff, S Shadomy, PF Troke, TJ Walsh and DW Warnock. Standardized testing of fluconazole: an International Collaborative Study. Antimicrobial Agents and Chemotherapy 1992, 36(9), 1805-1809. 